\title{
Minority stress, depression, and cigarette smoking among Chinese gay versus bisexual men: A two- group structural equation model analyses
}

Jingjing Li

Emory University School of Public Health https://orcid.org/0000-0003-4652-3379

Danqin Huang

Wuhan University, School of Health Sciences

Michael Windle

Emory University School of Public Health

Cam Escoffery

Emory University School of Public Health

Wei Wang

Xuzhou Medical University

Xiaoyan Li

Wuhan University School of Health Sciences

Kevin Tao

Georgia Institute of Technology College of Engineering

Regine Haardörfer

Emory University School of Public Health

Shiyue Li

Wuhan University School of Health Sciences

Carla J Berg

George Washington University Milken Institute of Public Health

Hong Yan ( $\nabla$ yanhmjxr@aliyun.com )

\section{Research article}

Keywords: Minority stress; depression; cigarette; smoking; gay men; bisexual men; China

Posted Date: November 4th, 2020

DOI: https://doi.org/10.21203/rs.2.17811/v4

License: (c) (i) This work is licensed under a Creative Commons Attribution 4.0 International License.

Read Full License 
Version of Record: A version of this preprint was published at BMC Public Health on July 9th, 2021. See the published version at https://doi.org/10.1186/s12889-021-10888-5. 


\section{Abstract}

Background: Literature in the West suggested that bisexual men have a higher smoking rate compared to gay men. Data on patterns of smoking among gay and bisexual men are limited in Eastern Asian countries like China. This study examined the cigarette smoking prevalence for gay versus bisexual men in China and their unique minority stress - smoking pathways.

Methods: Between September 2017 and November 2018, we surveyed a convenience sample of 538 gay men and 138 bisexual men recruited from local sexual minority organizations in four metropolitan cities in China (i.e., Beijing, Wuhan, Nanchang, and Changsha). Measures included sexual orientation, sociodemographics, theory-based minority stressors, depressive symptoms, and past 30-day cigarette smoking. Two-group (gay men vs. bisexual men) structural equation modeling (SEM) was used to test possible distinct mechanisms between theory-based stressors, depressive symptoms, and cigarette smoking among gay men and bisexual men, respectively.

Results: The mean age of participants was $26.51(\mathrm{SD}=8.41$ ) years old and $76.3 \%$ of them had at least a college degree. Bisexual men reported a higher rate of cigarette smoking compared to gay men (39.9\% vs. 27.3\%). Two-group SEM indicated that the pathways for cigarette smoking were not different between gay and bisexual men. Higher rejection anticipation was associated with greater depressive symptoms (standardized $\beta=0.32, p<.001$ ), and depressive symptoms were not associated with cigarette smoking.

Conclusions: Minority stress, specifically rejection anticipation, may be critical considerations in addressing depressive symptoms, but not smoking, among both gay and bisexual men in China.

\section{Background}

China has one of the highest burden of smoking in the world [1]. Each year, approximately 1,000,000 people in China die prematurely from smoking-related diseases [2, 3]. Men represent a disproportionate number of China's smokers. According to the 2015 China Adult Tobacco Survey, on average, $51.4 \%$ Chinese adult (aged 15 and older) men were current cigarette smokers, while $2.7 \%$ of adult women smoke [4]. While several Chinese metropolitan cities have passed increasingly progressive municipal-level tobacco control regulations, the prevalence of males who currently smoke cigarettes in these localities is still high, ranging from $32.7 \%$ to $44.5 \%$ [5].

Data from Canada, the U.S. and Australia has extensively studied tobacco use among sexual minority populations - or lesbians, gays, and bisexuals (LGB). This literature has largely indicated high rates of cigarette use among sexual minorities compared to the general populations [6-12]. While the literature regarding smoking among sexual minorities in Eastern Asian countries like China is limited, some studies have similarly shown increased smoking prevalence in this population. A 2015 study conducted in Shanghai, China found that gay and bisexual boys were more likely to smoke cigarette compared to heterosexual boys $(\mathrm{OR}=2.2)$, while lesbian and bisexual girls reported less any prior cigarette smoking compared to heterosexual girls $(\mathrm{OR}=0.7)$ [13]. Another study focusing on Chinese adult gay and bisexual 
men indicated a cigarette smoking prevalence of $66 \%$, [14] approximately $15 \%$ higher than the national average and $22 \%-33 \%$ higher than the city-level prevalence [5]. Gay and bisexual men represent a large subgroup in China. It was estimated that the total population of Chinese gay and bisexual men is around 18 (10.2-25.4) million [15]. However, limited research has focused on the heightened smoking rates among this large population of gay and bisexual men in China.

Minority Stress Theory (Meyer, 1995; 2003) is a framework for examining and understanding mechanism of high-risk behaviors in sexual minorities. Specifically, Minority Stress Theory views hostile social conditions as causes of stress for sexual minority groups $[16,17]$. Meyer $(1995 ; 2003)$ suggested that minority stress can take the forms of distal minority stress, or external processes and experiences faced by sexual minorities, including discrimination experiences; and proximal stress, or internal processes and experiences such as outness, rejection anticipation, identity concealment, and internalized homophobia $[16,17]$. These stressors can exacerbate mental health problems and consequently lead to increased health risk behaviors $[18,19]$. Empirically, numerous studies have shown that these minority stressors are positively associated with psychological distress and health risk behaviors such as tobacco use [9, 20-24]. For example, Burgess et al. (2008) pointed out that distal stressors such as discrimination experience was associated with greater likelihood of depression, anxiety, greater perceived needs for mental healthcare, and more frequent use of mental health services [25]. Others focusing on proximal stressors like internalized homophobia or outness showed that these stressors were signification predictors of mental health problems [26-29]. However, few studies have focused on minor frustrations and annoyance such as everyday discrimination that could produce long-lasting feelings of rejection [30], and one main reason for sexual minorities to use substance including cigarettes was the feeling of rejection [31]. As multiple stressors increasingly challenge an individual's coping capabilities, substance use such as smoking may serve as a coping strategy for sexual minorities who experience "minority stress" [10,16]. Conversely, positive coping such as resilience and coping resources such as social support were found to buffer the negative effects of minority stress on adverse health outcomes [32-36].

Contextual factors such as culture can be specific manifestation of Minority Stress Theory. Researchers suggested that Chinese culture is more collectivism that weights interpersonal bonds over individual themselves and emphasized a greater responsibility to the collective goods [37]. Lai (2010) and others believe part of the Chinese culture is influenced by Confucianism, a Chinese ancient philosophy that strongly emphasizes duty, obedience, filial piety, and certain ethical and moral values [38,39]. In research on Chinese contemporary sex norms, $\mathrm{Li}$ (2006) outlined that, cultivated in a collective culture, Chinese society sees the most commonly conducted behaviors as meeting the moral standards while less frequent behaviors would be viewed as 'abnormal' and 'unethical' [40]. Same-sex marriage is still illegal in China [41]. As a result, a sexual minority who does not act like his or her peers by not getting married at a certain age will become a concern and shame to the whole family for being deviant from the majority, and for failing to continue the family line [42]. Wang et al. (2015) found that gay/bisexual men in a qualitative study described constant pressure from family, social, and workplace to have a female partner, and they also stated the difficulties to establish stable same-sex relationships [43]. In 2020, researchers qualitatively examined minority stress among 24 Chinese sexual minority men and found that all 
participants suggested that Chinese culture (and cultural norm) functioned as source and context of minority stress [44]. Earlier study on Asian sexual minorities found same-sex behaviors would only be tolerant when they fulfill family duties including eventual heterosexual marriage [45]. In China, if men get older but are not married, family members, coworkers, peers and community acquaintances would start inquiring about their reasons for not getting married and even consider them as abnormal [46].

To date, the majority of research examining tobacco use through the lens of Minority Stress Theory has been done in North America $[6,47,48]$. In Southeast Asian countries, Minority Stress Theory was also used to inform study examining depression, suicide, and addictive behaviors among sexual minorities in Indonesia, Malaysia, Myanmar, Thailand, and Vietnam [49]. In Eastern Asian countries like China, the Minority Stress Theory has been almost exclusively applied to predicting physiological outcomes [50-53]. Although few Chinese studies have explicitly examined the mechanism between minority stress and cigarette use $[13,14,54]$, qualitative research has suggested that Chinese sexual minority men may use substances to relieve the stress of hostile social stigma, as well as familial and cultural pressures [55]. Therefore, it is important to empirically examine specific pathways through which minority stressors might influence cigarette use among Chinese sexual minorities, particularly gay and bisexual men.

Particularly relevant to the current study, not all sexual minorities experience the same stressors or related health risks. In North America, evidence across studies indicates significant inter-group difference across sexual minority subgroups that bisexuals are at increased risk for mental health problems and substance use compared to monosexuals (i.e., gay/lesbians and heterosexuals) $[47,56]$. Such intergroup differences between bisexuals and monosexuals can be explained by the unique minority stressors that bisexuals experience. For instance, bisexually-identified individuals might often be assumed to be gay/lesbian or heterosexual depending on the sex of their partners [56]. Thus, bisexual people might anticipate others to dismiss their bisexual identity; this anticipation may contribute to depressive symptoms and mental distress $[56,57]$. Other research by Sweet and Welles (2012) indicates that bisexual men may experience significantly more adverse childhood experiences compared to gay men [58]. However, in contrast to the growing body of literature examining the heterogeneity of sexual minority stressors and subsequent psychological and health behavior risks across sexual minority subgroups in North America, very little is known about this topic in China. One 2015 paper by Lian et al. in China studied smoking among sexual minority youth and actually found the reverse pattern, that male bisexual and heterosexual youth shared similar smoking patterns while gay youth reported higher smoking rates compared to heterosexual counterparts [13]. Lian and colleagues believed that bisexual youth in China might be less involved with LGB activities with higher risk for smoking exposure (e.g., pride events), compared to gay youth; or, bisexual youth in China might be less likely to cope minority stress with smoking [13].

To date, it is unclear whether significant heterogeneity exist between Chinese gay and bisexual men regarding their minority stress experiences and related health risks. This study aimed to contribute to the literature by examining the prevalence of cigarette smoking in a sample of Chinese gay and bisexual men and their distinct minority stress - smoking pathways, using the two-group (gay vs. bisexual men) structural equation modeling (SEM). This research is critical, as the exploration of minority stressors and 
pathways among subgroups of sexual minority men may help advance our understanding of cigarette use behavior among high-risk populations in China and develop effective tobacco cessation interventions.

\section{Methods}

\section{Study Population and Data Collection}

The authors obtained data from a survey study focusing on health risk behaviors of Chinese sexual minority men conducted by School of Health Sciences, Wuhan University, China. Details of the survey methodology have been published elsewhere [58]. Briefly, between September 2017 and January 2018, we collected baseline survey data of 755 self-identified gay and bisexual men using venue-based sampling from college campus-based sexual minority-serving organizations in four cities, namely Beijing, Wuhan, Nanchang, and Changsha, as these cities have high concentration of LGBT populations. These data are among the most recent data available regarding tobacco use among sexual minority men in China. This convenient sample was mainly comprised of urban, well-educated, and high-income gay/bisexual men, and might not be representative to all sexual minority men. After screening for eligibility (self-identified as gay/bisexual men and aged 16 years or older), participants were given a brief explanation of the survey's purpose. Informed consent was obtained from every participant. Each participant received a one-time compensation of 50 Chinese RMB (approximately \$8 US dollars) for their time. For this study, we excluded 79 (10.5\%) participants who reported "heterosexual/unsure/other" sexual orientation. Participants involved in tobacco cessation programs, including HIV-positive individuals who were linked to HIV care and provided with behavioral intervention (i.e., tobacco cessation services) [59], were excluded from this study. The analytical sample size for this study was 676 . This study was approved by the Institutional Review Board (IRB) at [blinded for review].

\section{Measures}

Primary outcome: Cigarette smoking. Participants were asked, "During the past 30 days, on how many days did you smoke cigarettes? Little cigars or cigarillos? Traditional pipe? Chewing tobacco? Ecigarettes? Hookah?" Given that fewer than 10 participants reported on alternative tobacco use (i.e., little cigars or cigarillos, traditional pipe, chewing tobacco, e-cigarette, and hookah), this study only focused on the past 30-day cigarette smoking outcome, dichotomized as "no" versus "any smoking."

Primary grouping variable: Sexual orientation. Sexual orientation was assessed by asking, "Is your sexual orientation: heterosexual; gay or homosexual; bisexual; or unsure?" All responses were coded dichotomously $(0=$ gay and $1=$ bisexual).

Sociodemographic characteristics. Participants were asked to provide sociodemographic information, including age, education (high school/below vs. college/above), place of origin (urban vs. rural), employment (dummy coded into student, employed, and unemployed), marital status (unmarried/divorced vs. married), monthly income ( $\leq 3000 \mathrm{RMB}$ [73 USD] vs. > $3000 \mathrm{RMB}$, note that the minimum monthly 
wage ranges between 1580 and 2000 RMB in 4 sampled cities [60]), and nature of their health insurance (yes vs. no/unsure).

Depressive symptoms as mediator. Depressive symptoms were hypothesized as a mediator of the relationships between minority stressors and cigarette smoking (Figure 1) and were assessed with the Center for Epidemiological Studies Depression (CES-D) scale. [61] The CES-D is a well-established and widely used [62] 20-item scale designed to measure depressive symptoms experienced by the individual within the past week, and its Chinese version has been validated [63, 64]. Items were answered on a 4point scale ranging from $0=$ less than a day or never to $3=5-7$ days. The Cronbach's alpha was 0.89 .

Minority Stressors. Informed by Minority Stress Theory [16, 17], we measured distal minority stressors (everyday discrimination), proximal minority stressors (outness, rejection anticipation, identity concealment, and internalized homophobia), general stressors (adverse childhood experiences), and stress-moderating factors (social support and resilience).

Everyday discrimination was assessed using the Everyday Discrimination Scale [65]. This scale asks about the frequency of 9 types of hassles and prejudice events that sexual minority people may encounter. This 9-item scale is rated on a 6-point Likert scale with response options of 1=happens daily to 6=never happened. All responses were reverse coded and averaged to create a mean score, with higher scores indicating severer everyday discrimination. The Cronbach's alpha coefficient was 0.94 for this scale.

Outness was assessed by asking respondents "Have you ever 'come out' to anyone?" All responses were coded dichotomously $(0=$ no and $1=y e s)$.

Rejection anticipation was assessed with a scale which was originally used to assess stigma of mental illness [66]. Later, this scale was modified and adapted by Forst et al. (2015) for assessing sexual minority's state of hypervigilance and worry about being rejected.[21] This 6-item scale is rated on a 5point Likert scale, ranging from $1=$ applies very strongly to $5=$ does not apply at all. All responses were reverse coded and averaged to create a mean score, with higher scores indicating higher expectation of rejection. The Cronbach's alpha of this scale in this study was 0.88 .

Identity concealment was assessed using a subscale on nondisclosure developed and validated by Testa et al.(2015) [67]. This 6-item scale asks about the intentions and behaviors of sexual minority individuals to avoid disclose their sexual minority identities. This scale is rated on a 5-point Likert scale, ranging from $1=$ applies very strongly to $5=$ does not apply at all. All responses were reverse coded and averaged to create a mean score, with higher scores indicating greater identity concealment. The Cronbach's alpha was 0.91 .

Internalized homophobia was assessed using the Internalized Homophobia Scale which was originally developed by Martin and Dean (1987) [68] and further modified and validated by Forst et al. $(2009 ; 2015)$. $[21,69]$ This scale asks about the negative attitudes sexual minorities hold against their own sexual identities. This 8-item scale is rated on a 4-point Likert scale, ranging from $1=n e v e r$ to $4=a /$ ways. 
Responses were averaged to create a mean score, with higher scores indicating greater internal homophobia. The Cronbach's alpha was 0.89 .

Adverse childhood experiences (ACES) was assessed using a 10-item ACEs index developed by the U.S. Centers for Disease Control and Prevention [70,71]. This index asks about the physical and mental abuse and traumatic experiences of participants prior to 18 years old. Participants answered $0=n o$ or $1=y e s$ to each item. Total score of all responses was summed; higher scores indicate more ACEs. The Cronbach's alpha was 0.63 .

Social support was assessed with the Multidimensional Scale of Perceived Social Support,[72] which measures perceived support from family, friends, and significant others. This 12 -item scale is rated on a 7point Likert scale, ranging from $1=$ very strongly disagree to $7=$ very strongly agree. The total score of this scale was calculated for each participant, with higher scores indicating more social support. The Cronbach's alpha was 0.94 .

Resilience was assessed with the 10-item Connor-Davidson Resilience scale.[73, 74] This scale is a measure of stress coping capabilities. It is rated on a 5 -point Likert scale ranging from $1=$ not true at all true to $5=$ true nearly all of the time. The responses are summed to derive a total score, with higher scores indicating more resilience. The Cronbach's alpha was 0.95 .

\section{Statistical Analysis}

Univariate analyses were conducted to examine the distribution of each variable. ANOVA and chi-square tests were conducted to assess the bivariate relationships between sociodemographic and psychosocial variables and the cigarette smoking outcome. We also examined the multi-collinearity between all variables. In consideration of study power, we excluded selected sociodemographic variables (i.e., education, marriage, monthly income, and health insurance) from the modeling analyses, as these variables either were not associated with the outcome or showed considerable collinearity (data not shown).

Next, we conducted sequential logistic regressions to identify significant associations between predictors and cigarette use to inform the approach to the structural equation models (SEMs). Specifically, we assessed the effects of sexual orientation on cigarette use in following models: (1) only included sexual orientation as predictor; (2) added other sociodemographic predictors (i.e., age, place of origin, and employment); (3) added psychosocial risk factors (i.e., everyday discrimination, outness, rejection anticipation, identity concealment, internalized homophobia, and ACEs), and (4) added psychosocial protective factors (i.e., social support and resilience).

For the two-group SEM, we specified the SEM model in Figure 2 based on Minority Stress Theory, preliminary analyses, and correlation matrix results. SEM is a process that allows for testing one or more theories that are hypothesized a prior to explain the characteristics of measured variables [59]. SEM can be used for model confirmatory purpose, testing alternative models, or model generation [60]. Two-group SEM can be used to examine inter-group differences across sexual minority subgroups with increased 
rigor. The advantage of two-group SEM is that it allows the comparison of the extent of associations based on path coefficients [61] and uses model fit indices to determine which tested paths best fit the data. This method could help examine whether the underlying pathway are significantly different among sexual minority subgroups. Given the goals of this study, two-group SEM is a particularly useful tool for examining how different pathways might vary across sexual orientation. A two-phase modeling approach was used for the two-group SEM. First, we examined measurement invariance (i.e., item-scale relationships) between gay and bisexual participants using confirmatory factor analysis (CFA). Second, we examined structural invariance (i.e., hypothesized relationships among variables) between gay and bisexual participants. Chi-square differences between these two models were examined and indicated non-significant results and thus no group differences in the measurement models [75].

Then, we examined the model fit and path-coefficients of the final two-group structural SEM. The model fit indices included: chi-square test, standardized root mean square residual (SRMR), comparative fit index (CFI), Tucker-Lewis fit index (TLI), the root mean-square error of approximation (RMSEA), and weighted root mean square residual (WRMR). The indicators of goodness of fit were: $\chi 2 p<0.05$; SRMR $>0.08 ; C F I>$ 0.90; TLI > 0.90; RMSEA < 0.05; and WRMR < 1 [76]. The bootstrapping method was used to test $95 \%$ Confidence Interval $(95 \% \mathrm{Cl})$. Standardized regression $(\beta)$ coefficients, the standard errors, and $p$-values for $\beta$ were reported in the final model.

Data were double-entered and cleaned using EpiData 3.1 (The EpiData Association, Odense, Denmark) software. Descriptive analysis and sequential regressions were conducted using SAS 9.4 (SAS Institute Inc.: Cary, NC, USA). Two-group SEM was conducted using Mplus 8.0 (Muthén \& Muthén: Los Angeles, CA, USA).

\section{Results}

\section{Descriptive Statistics}

Table 1 shows that, among the full sample, the proportion of cigarette use was $29.9 \%$. The mean age of the participants was 26.51 (SD $=8.41$ ) years old, $76.3 \%$ reported holding college degree or above, $31.5 \%$ were current students, and $58.6 \%$ were employed. Bisexual men reported a higher rate of cigarette smoking compared to gay men (39.9\% vs. $27.3 \%$ ). Rejection anticipation was significantly associated with cigarette smoking. Because marriage and health insurance were not associated with cigarette smoking, these variables were excluded from subsequent modeling analyses. Because education and monthly income correlated with employment, we included employment but excluded education and monthly income in the subsequent modeling.

Table 1. Descriptive characteristics and bivariate analyses examining differences between past 30-day cigarette smoking $(\mathrm{N}=202)$ vs. non-smoking $(\mathrm{N}=474)$ in Chinese gay and bisexual men $(\mathrm{N}=676)$ 


\begin{tabular}{|c|c|c|c|c|}
\hline \multirow[b]{2}{*}{ Variables } & \multirow{2}{*}{$\begin{array}{c}\text { Total } \\
\text { Sample } \\
\text { M (SD) or N (\%) } \\
\text { N=676 }\end{array}$} & \multicolumn{2}{|c|}{ Smoking Status } & \multirow[b]{2}{*}{$p$} \\
\hline & & $\begin{array}{c}\text { Smoking } \\
\mathrm{M}(\mathrm{SD}) \text { or } \mathrm{N}(\%) \\
\mathrm{N}=202\end{array}$ & $\begin{array}{c}\text { Nonsmoking } \\
M(\mathrm{SD}) \text { or } \mathrm{N}(\%) \\
\mathrm{N}=474\end{array}$ & \\
\hline \multicolumn{5}{|l|}{ Sexual Orientation (\%) } \\
\hline Gay & $538(79.6)$ & $147(72.8)$ & $391(82.5)$ & .004 \\
\hline Bisexual & $138(20.4)$ & $55(27.2)$ & $83(17.5)$ & \\
\hline \multicolumn{5}{|l|}{ Sociodemographics } \\
\hline Age (SD) & $26.51(8.41)$ & $27.33(9.35)$ & $26.16(7.95)$ & .102 \\
\hline \multicolumn{5}{|l|}{ Education (\%) } \\
\hline High school or below & $160(23.7 \%)$ & $72(35.6)$ & $88(18.6)$ & $<.001$ \\
\hline College or above & $516(76.3 \%)$ & $130(64.4)$ & $386(81.4)$ & \\
\hline \multicolumn{5}{|l|}{ Place of Origin (\%) } \\
\hline Urban & $412(61.0)$ & $118(58.4)$ & $294(62.0)$ & .379 \\
\hline Rural & $264(39.0)$ & $84(41.6)$ & $180(38.0)$ & \\
\hline \multicolumn{5}{|l|}{ Employment (\%) } \\
\hline Student & $213(31.5)$ & $40(19.8)$ & $173(36.5)$ & $<.001$ \\
\hline Employed & $396(58.6)$ & $131(64.9)$ & $265(55.9)$ & \\
\hline Unemployed & $67(9.9)$ & $31(15.3)$ & $36(7.6)$ & \\
\hline \multicolumn{5}{|l|}{ Marital Status (\%) } \\
\hline Unmarried/divorced & $602(89.1)$ & $181(89.6)$ & $421(88.8)$ & .764 \\
\hline Married & $74(10.9)$ & $21(10.4)$ & $53(11.2)$ & \\
\hline \multicolumn{5}{|l|}{ Monthly Income in RMB (\%) } \\
\hline$\leq 3000(73$ USD $)$ & $352(52.1)$ & $93(46.1)$ & $259(54.6)$ & .040 \\
\hline$>3000$ & $324(47.9)$ & $109(53.9)$ & $215(45.4)$ & \\
\hline \multicolumn{5}{|l|}{ Health Insurance (\%) } \\
\hline Yes & $565(83.6)$ & $161(79.7)$ & $404(85.2)$ & .076 \\
\hline No/Unsure & $111(16.4)$ & $41(20.3)$ & $70(14.8)$ & \\
\hline \multicolumn{5}{|l|}{ Minority Stressors } \\
\hline Everyday Discrimination (SD) & $2.06(1.11)$ & $2.10(1.15)$ & $2.05(1.10)$ & .583 \\
\hline \multicolumn{5}{|l|}{ Outness (\%) } \\
\hline Yes/ever coming out & $549(81.2)$ & $156(77.2)$ & $393(82.9)$ & .083 \\
\hline Never & $127(18.8)$ & $46(22.8)$ & $81(17.1)$ & \\
\hline Rejection Anticipation (SD) & $2.44(1.02)$ & $2.29(0.95)$ & $2.49(1.04)$ & .013 \\
\hline Identity Concealment (SD) & $2.80(1.09)$ & $2.84(1.07)$ & $2.78(1.09)$ & .527 \\
\hline Internal Homophobia (SD) & $1.63(0.68)$ & $1.77(0.71)$ & $1.66(0.67)$ & .058 \\
\hline ACEs (SD) & $1.00(1.38)$ & $1.12(1.33)$ & $0.96(1.39)$ & .183 \\
\hline Depressive symptoms (SD) & $17.47(10.59)$ & $17.75(10.74)$ & $17.34(10.54)$ & .643 \\
\hline Social Support (SD) & $5.07(1.02)$ & $4.99(1.09)$ & $5.11(0.99)$ & .190 \\
\hline Resilience (SD) & $26.71(8.45)$ & $26.89(8.45)$ & $26.64(8.38)$ & .724 \\
\hline
\end{tabular}

\section{Correlation Statistics}


The correlation matrix examining group differences between gays and bisexuals (Table 2) indicated that employment was the only significant correlate of cigarette smoking in both gay and bisexual subgroups. Among all psychosocial factors, group differences were only found regarding rejection anticipation and depressive symptoms. Thus, the two-group SEM included rejection anticipation, depressive symptoms, and cigarette smoking, predicted by employment (dummy coded as student or employed) (Figure 2).

Table 2. Correlation matrix examining correlates of past 30-day cigarettes smoking among the full sample of Chinese sexual minority men, gays, and bisexuals, respectively $(\mathrm{N}=676)$

\begin{tabular}{|c|c|c|c|}
\hline Variables & $\begin{array}{c}\text { Total sample } \\
\text { r coefficient, } \\
p \text { value } \\
\mathrm{N}=676\end{array}$ & $\begin{array}{c}\text { Gay } \\
\text { r coefficient, } \\
p \text { value } \\
\mathbf{N}=538 \\
\end{array}$ & $\begin{array}{c}\text { Bisexual } \\
\text { r coefficient, } \\
p \text { value } \\
\mathrm{N}=138 \\
\end{array}$ \\
\hline \multicolumn{4}{|l|}{ Sociodemographics } \\
\hline Age & $\begin{array}{c}0.063 \\
.102\end{array}$ & $\begin{array}{c}0.061 \\
.161\end{array}$ & $\begin{array}{c}-0.005 \\
.949\end{array}$ \\
\hline Place of Origin & $\begin{array}{c}0.034 \\
.379\end{array}$ & $\begin{array}{c}0.033 \\
.448\end{array}$ & $\begin{array}{c}0.008 \\
.920\end{array}$ \\
\hline Employment & $\begin{array}{l}0.185 \\
<.001\end{array}$ & $\begin{array}{l}0.157 \\
<.001\end{array}$ & $\begin{array}{l}0.240 \\
0.004\end{array}$ \\
\hline \multicolumn{4}{|l|}{ Minority Stressors } \\
\hline Everyday Discrimination & $\begin{array}{c}0.021 \\
.583\end{array}$ & $\begin{array}{c}0.030 \\
.481\end{array}$ & $\begin{array}{c}-0.032 \\
.710\end{array}$ \\
\hline Outness & $\begin{array}{c}0.067 \\
.084\end{array}$ & $\begin{array}{c}0.023 \\
.591\end{array}$ & $\begin{array}{c}0.068 \\
.423\end{array}$ \\
\hline Rejection Anticipation & $\begin{array}{c}-0.095 \\
.013\end{array}$ & $\begin{array}{c}-0.102 \\
.018\end{array}$ & $\begin{array}{c}-0.066 \\
.444\end{array}$ \\
\hline Identity Concealment & $\begin{array}{c}0.024 \\
.526\end{array}$ & $\begin{array}{c}0.029 \\
.497\end{array}$ & $\begin{array}{c}-0.015 \\
.864\end{array}$ \\
\hline Internal Homophobia & $\begin{array}{c}0.073 \\
.058\end{array}$ & $\begin{array}{c}0.005 \\
.900\end{array}$ & $\begin{array}{r}0.129 \\
.129\end{array}$ \\
\hline ACEs & $\begin{array}{c}0.054 \\
.183\end{array}$ & $\begin{array}{c}0.054 \\
.232\end{array}$ & $\begin{array}{c}0.082 \\
.378\end{array}$ \\
\hline Depressive symptoms & $\begin{array}{c}0.018 \\
.643\end{array}$ & $\begin{array}{c}-0.032 \\
.459\end{array}$ & $\begin{array}{r}0.199 \\
.019\end{array}$ \\
\hline Social support & $\begin{array}{c}-0.050 \\
.190\end{array}$ & $\begin{array}{c}-0.046 \\
.291\end{array}$ & $\begin{array}{c}-0.051 \\
.550\end{array}$ \\
\hline Resilience & $\begin{array}{c}0.013 \\
.723\end{array}$ & $\begin{array}{c}0.024 \\
.575\end{array}$ & $\begin{array}{c}-0.013 \\
.882\end{array}$ \\
\hline
\end{tabular}

\section{Sequential Logistic Regression Models}

Table 3 shows the results from four regression models. Model 1 result showed that compared to gay men, bisexual men were more likely to use cigarette (crude Odd Ratio $[\mathrm{OR}]=1.76,95 \% \mathrm{Cl}$ : $1.19-2.60$ ). In model 2, bisexual men were still more likely to use cigarette compared to gay men (adjust $\mathrm{OR}=1.79 ; 95 \% \mathrm{Cl}: 1.18$ - 2.72). Compared to student, being unemployed was more likely to use cigarette $(\mathrm{aOR}=3.96 ; 95 \% \mathrm{Cl}$ : 2.09 - 7.50). In model 3, only being unemployed (aOR: 3.69; 95\% Cl: 1.85 - 7.37) and rejection anticipation (aOR: 0.80; 95\% Cl: $0.65-1.00$ ) were associated with cigarette use. In model 4, being unemployed was the only significant correlate of cigarette use (aOR: $3.78 ; 95 \% \mathrm{Cl}: 1.90-7.54)$. The majority of psychosocial factors were not associated with the outcome. 
Table 3. Sequential logistic regressions identifying correlates of past 30-day cigarette use among gay and bisexual men in China $(\mathrm{N}=676)$

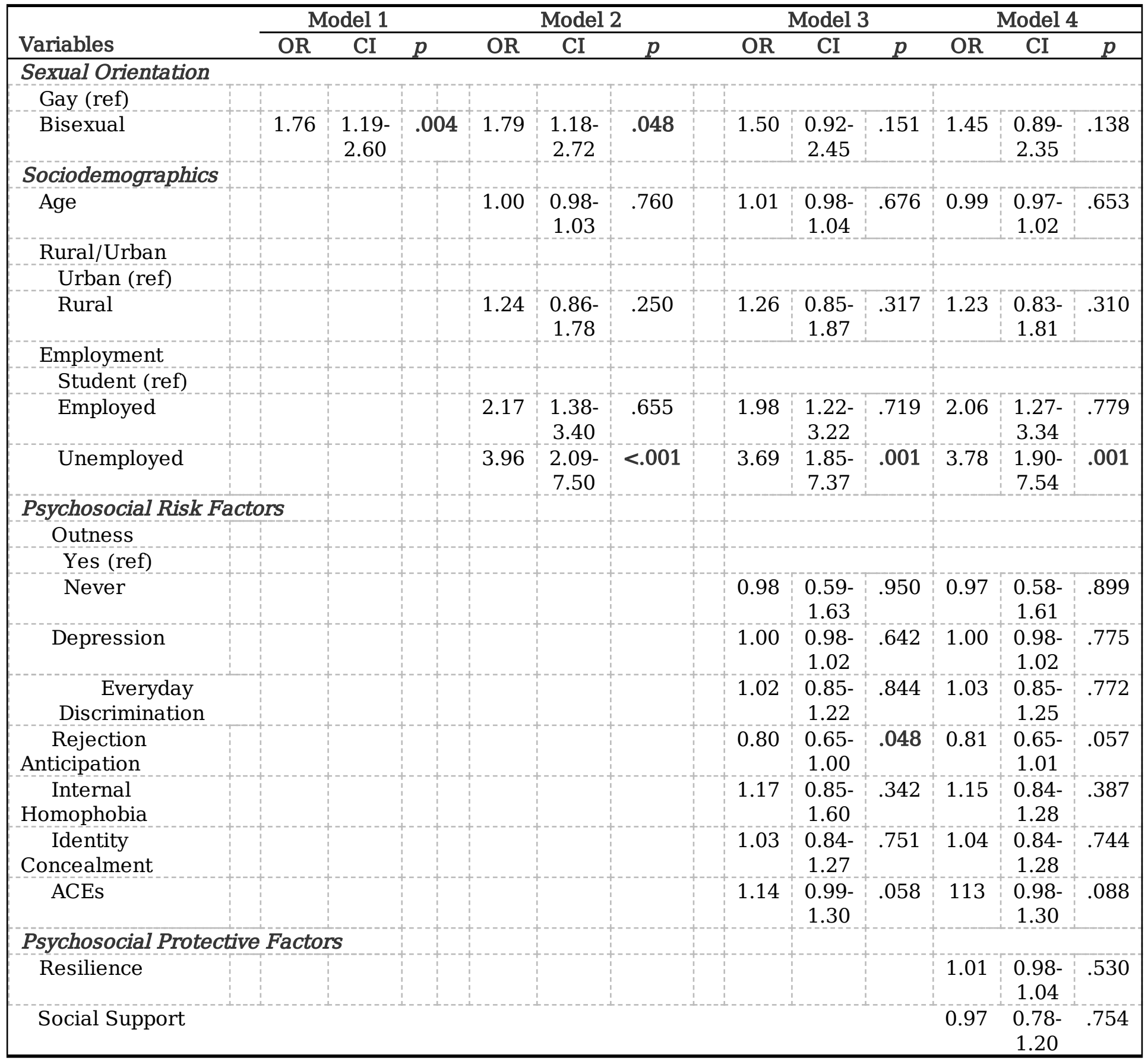

\section{Two-Group SEM}

Measurement model. We constructed unconstrained and constrained measurement CFA models. According to the model fit indices for the unconstrained model $(\chi 2=1326.7$ (576), $p<.001$; RMSEA $=.061$; $\mathrm{CFI}=.908 ; \mathrm{TLI}=.896 ; \mathrm{SRMR}=.056)$ and factor-loading constrained model $(\mathrm{X} 2=1358.2(600), \mathrm{p}<.001$; RMSEA $=.061 ; \mathrm{CFI}=.907 ; \mathrm{TLI}=.900 ;$ SRMR $=.061)$, the chi-square difference test $(X 2=31.5[24])$ was nonsignificant, indicating factor loading invariance. Therefore, we moved on to the structural analysis using this invariant measurement model. 
Structural model. According to the model fit indices for the unconstrained model $(\chi 2=971.0(761), p<.001$; RMSEA $=.029 ; \mathrm{CFI}=.920 ; \mathrm{TLI}=.916 ; \mathrm{WRMR}=1.312)$ and constrained model $(X 2=981.3(770), \mathrm{p}<.001 ;$ RMSEA $=.028 ; \mathrm{CFI}=.920 ; \mathrm{TLI}=.916 ; \mathrm{WRMR}=1.397)$, the chi-square difference test $(X 2=10.3[9])$ was non-significant, indicating structural invariance for gay and bisexual samples. The final two-group SEM fits the data well.

Final modeling results. For both gay and bisexual men (Figure 3), rejection anticipation was positively associated with greater depressive symptoms (standardized $\beta=0.30, p<.001$ ) and negatively associated with being a current cigarette smoker (standardized $\beta=-0.15, p<.001$ ). Being a student was positively associated with higher rejection anticipation (standardized $\beta=0.17, p<.05$ ) and was negatively associated with cigarette smoking (standardized $\beta=-0.33, p<.05$ ). Being a student was not associated with depressive symptoms. Depressive symptoms were not associated with cigarette smoking.

\section{Discussion}

This study examined cigarette smoking among sexual minority men in China through the lens of the Minority Stress Theory. Our findings showed that bisexual men reported a higher rate of cigarette smoking compared to gay men (39.9\% vs. $27.3 \%)$ in China. This is consistent with literature in other countries that bisexual men often report higher use of cigarette relative to gay men $[77,78]$.

Minority stress, particularly rejection anticipation, was positively associated with depressive symptoms in both gay and bisexual subgroups. This finding is consistent with studies suggesting that rejection anticipation is associated with the onset of depressive symptoms $[79,80]$. In Chinese culture, being a majority represents righteousness and power, whereas minorities may be marginalized and/or ostracized [40]. Rejection anticipation was reversely associated with cigarette smoking. This unexpected result might be influenced by the launch of public smoke-free legislation in major cities like Beijing since 2014, that participants who anticipated rejections based on their sexual minority orientations might tend to minimize other types of rejections from the society including smoking. Taken together, rejection anticipation is a critical construct for sexual minority stress that might predict important mental health outcomes, especially in a collective culture like China. We did not find associations between other minority stressors and depressive symptoms. However, a study in Thailand suggested that experiences of victimization, discrimination, and identify concealment also predicted depression among sexual minorities [81]. More research is needed to confirm the relationship between various minority stressors and depressive symptoms in different cultural contexts.

No direct relationship between depression and cigarette smoking among gay and bisexual men was indicated. However, other research has documented significant relationships between mental distress and smoking among sexual minority men globally [29, 82-84]. There might be some contextual factors - such as tobacco-free policy in our campus-based venues, peer influences, and social or cultural norms confounding or buffering the effect of minority stressors on cigarette use in this study population. For example, structural discrimination was found to be a significant predictor of smoking among sexual minority subgroup [85]. Peer violence and pressure also could lead to tobacco use among sexual minority 
youth [86]. Future studies should consider the contextual influencing factors to better understand the effects of minority stressors on cigarette smoking.

We did not find distinctive inter-group differences between subgroups in the mechanism through which minority stress might affect cigarette smoking. According to a Chinese qualitative study on bisexuality, bisexual identity is a fairly new concept for many Chinese, especially among young adults and students, and bisexual individuals might undergo long time exploration of sexual identity and might experience enormous confusion and social pressure [87]. It may be that some young participants or student participants in our study might still be exploring their identities and assume themselves to be gay, bisexual, or heterosexual, depending on the sex of their current partners [56]. Thus, the distinction between gay men and bisexual men might be biased because of high proportion of students in our study. More studies are needed to explain why bisexual men experienced higher cigarette smoking in China.

Results indicated that being a student was positively associated with rejection anticipation among Chinese sexual minority men. Currently, no known study has evaluated the impact of school environments or policies on the health of sexual minority students in China, but empirical studies in the U.S. have found that sexual minority students experience higher rates of parental or peer rejections compared to heterosexual counterparts [88]. Moreover, $86.2 \%$ of U.S. sexual minority students experienced verbal harassment and $44.1 \%$ of these students experienced physical harassment [89]. In the U.S., sexual minority students felt less safe at school compared to heterosexual students [90]. Depending on the school climate, sexual minority students might be experiencing in a hostile or protective environment. Thus, more studies are needed to evaluate the impact of school setting toward their feeling of rejection, mental illness, and health risk behaviors among Chinese sexual minority students.

\section{Limitations}

We used data from a convenient sample that was mainly comprised of urban, well-educated, and highincome gay/bisexual men, which might not be representative to all sexual minority men. Thus, our study provided little evidence base regarding the mental health status and smoking behaviors among LGBs residing in rural areas of China or who are with lower socioeconomic statuses. Also, the data were collected in sexual minority-serving organizations located in or close to college campuses, thus our measurement of cigarette smoking might be influenced by the smoke-free campus policies enforced since 2014 and therefore might not reflect the actual cigarette smoking prevalence. The cross-sectional nature of the data limits the causal inference regarding our findings. Due to the page limitation of the survey instrument, we did not assess important factors that might influence smoking behaviors such as cultural aspects or peers or family influences. Last, data were collected based on self-report using pen-and-paper and thus might be prone to recall bias and social desirability.

\section{Conclusions}

Guided by Minority Stress Theory, this study is among the first to examine the minority stress - cigarette use pathway among a sample of Chinese gay and bisexual men. Our findings showed that bisexual men 
are more likely to smoke cigarette compared to gay men. Minority stress, particularly rejection anticipation, was associated with depressive symptoms in both gay and bisexual men, but depressive symptoms were not associated with cigarette smoking. Although we did not identify the pathway linking minority stress and cigarette smoking between gay and bisexual men, future studies can explore the role of minority stress and differences in cigarette smoking rates across sexual minority subgroups in urban and rural China and beyond in order to design tailored and targeted tobacco cessation interventions.

\section{List Of Abbreviations}

SEM: structural equation modeling

SD: standard deviation

OR: Odd Ratio

Cl: Confidence Interval

CES-D: Center for Epidemiological Studies Depression Scale

ACEs: Adverse childhood experiences

ANOVA: Analysis of variance

CFA: confirmatory factor analysis

SRMR: standardized root mean square residual

CFI: comparative fit index

TLI: Tucker-Lewis fit index

RMSEA: the root mean-square error of approximation

WRMR: weighted root mean square residual

\section{Declarations}

\section{Ethics approval and consent to participate}

Our project was approved by the Wuhan University Institutional Review Boards. All participants provide consent prior to completing the survey.

\section{Consent for Publication}

Written consent forms were obtained from all survey participants for publication. 
Availability of data and materials

The datasets used and/or analyzed during the current study are available from the corresponding author (Dr. Hong Yan) on reasonable request.

\section{Competing interests}

All authors declare no competing interests.

\section{Funding}

This research is supported by the Emory University Professional Development Funds (PI: JL), China Humanities and Social Science Foundation, Ministry of Education (15YJAZH094, PI: HY), and National Natural Science Foundation of China (81673196, PI: HY). The funders had no role in the study design, collection, analysis or interpretation of the data, writing the manuscript, or the decision to submit the paper for publication.

\section{Authors' contributions}

$\mathrm{JL}$ conceptualized the study, performed data analysis, and wrote the manuscript. DH contributed to the manuscript writing and manuscript review. CJB, MW, and $\mathrm{RH}$ contributed to the conceptualization of the study and oversaw data analytical methods. CE and CJB reviewed data analytical methods and contributed to manuscript writing. WW, XL, SL, and HY collected the data. KT, HY, and SL reviewed data analytical methods and contributed to manuscript writing. All authors have read and approved the manuscript.

\section{Acknowledgements}

We thank all the LGBT organizations in Beijing, Wuhan, Nanchang, and Changsha, China for their supports in our research data collection.

\section{References}

1. WHO report on the global tobacco epidemic, 2017 [https://www.who.int/tobacco/surveillance/policy/country_profile/chn.pdf?ua=1]

2. Hu SS: Tobacco product use among adults-United States, 2013-2014. MMWR Morbidity and Mortality Weekly Report 2016, 65.

3. Chen ZM, Peto R, lona A, Guo Y, Chen YP, Bian Z, Yang L, Zhang WY, Lu F, Chen JS: Emerging tobaccorelated cancer risks in China: A nationwide, prospective study of $\mathbf{0 . 5}$ million adults. Cancer 2015, 121(S17):3097-3106.

4. China CDC: 2015 China Adult Tobacco Survey [In Chinese]. In. Beijing, China: China CDC; 2015.

5. China CDC: Report of China City Adult Tobacco Survey 2013-14: A 14-city experience. In. UK: WHO; 2015. 
6. Wheldon CW, Kaufman AR, Kasza KA, Moser RP: Tobacco Use Among Adults by Sexual Orientation: Findings from the Population Assessment of Tobacco and Health Study. Lgbt Health 2018, 5(1):3344.

7. Baskerville NB, Dash D, Shuh A, Wong K, Abramowicz A, Yessis J, Kennedy RD: Tobacco use cessation interventions for lesbian, gay, bisexual, transgender and queer youth and young adults: A scoping review. Preventive medicine reports 2017, 6:53-62.

8. Berger I, Mooney-Somers J: Smoking cessation programs for lesbian, gay, bisexual, transgender, and intersex people: a content-based systematic review. Nicotine Tob Res 2016, 19(12):1408-1417.

9. Goldbach JT, Tanner-Smith EE, Bagwell M, Dunlap S: Minority Stress and Substance Use in Sexual Minority Adolescents: A Meta-analysis. Prev Sci 2014, 15(3):350-363.

10. Blosnich J, Lee JG, Horn K: A systematic review of the aetiology of tobacco disparities for sexual minorities. Tob Control 2013, 22(2):66-73.

11. Lee JG, Griffin GK, Melvin CL: Tobacco use among sexual minorities in the USA, 1987 to May 2007: a systematic review. Tob Contro/ 2009, 18(4):275-282.

12. Schauer GL, Berg CJ, Bryant LO: Sex differences in psychosocial correlates of concurrent substance use among heterosexual, homosexual and bisexual college students. The American journal of drug and alcohol abuse 2013, 39(4):252-258.

13. Lian Q, Zuo X, Lou C, Gao E, Cheng Y: Sexual orientation and smoking history: results from a community-based sample of youth in Shanghai, China. Environmental health and preventive medicine 2015, 20(3):179.

14. Berg CJ, Nehl EJ, Wong FY, He N, Huang ZJ, Ahluwalia JS, Zheng T: Prevalence and Correlates of Tobacco Use Among a Sample of MSM in Shanghai, China. Nicotine Tob Res 2011, 13(1):29-35.

15. Zhang BC, Li XF, Shi TX, Yang LG, Zhang JD: A rough estimate of gay/lesbian population and their infect HIV prevalence. Chin J STD/AIDS Prev Cont 2002, 8:197-199.

16. Meyer IH: Prejudice, social stress, and mental health in lesbian, gay, and bisexual populations: conceptual issues and research evidence. Psychol Bull 2003, 129(5):674.

17. Meyer IH: Minority stress and mental health in gay men. Journal of health and social behavior 1995:38-56.

18. Fluharty M, Taylor AE, Grabski M, Munafò MR: The association of cigarette smoking with depression and anxiety: a systematic review. Nicotine Tob Res 2016, 19(1):3-13.

19. Ziedonis D, Hitsman B, Beckham JC, Zvolensky M, Adler LE, Audrain-McGovern J, Breslau N, Brown RA, George TP, Williams J: Tobacco use and cessation in psychiatric disorders: National Institute of Mental Health report. In.: Society for Research on Nicotine and Tobacco; 2008.

20. Gamarel KE, Mereish EH, Manning D, Iwamoto M, Operario D, Nemoto T: Minority Stress, Smoking Patterns, and Cessation Attempts: Findings From a Community-Sample of Transgender Women in the San Francisco Bay Area. Nicotine Tob Res 2016, 18(3):306-313.

21. Frost DM, Lehavot $\mathrm{K}$, Meyer $\mathrm{IH}$ : Minority stress and physical health among sexual minority individuals. Journal of behavioral medicine 2015, 38(1):1-8. 
22. Wong CF, Schrager SM, Holloway IW, Meyer IH, Kipke MD: Minority stress experiences and psychological well-being: The impact of support from and connection to social networks within the Los Angeles house and ball communities. Prev Sci 2014, 15(1):44-55.

23. Lehavot K: Minority stress, coping, and health among sexual minority women. US: ProQuest Information \& Learning; 2012.

24. Hamilton CJ, Mahalik JR: Minority Stress, Masculinity, and Social Norms Predicting Gay Men's Health Risk Behaviors. J Couns Psychol 2009, 56(1):132-141.

25. Burgess D, Lee R, Tran A, Van Ryn M: Effects of perceived discrimination on mental health and mental health services utilization among gay, lesbian, bisexual and transgender persons. Journal of LGBT Health Research 2008, 3(4):1-14.

26. Newcomb ME, Mustanski B: Internalized homophobia and internalizing mental health problems: A meta-analytic review. Clinical psychology review 2010, 30(8):1019-1029.

27. Villarreal C, Wiebe JS, Sauceda JA, Simoni JM: Internalized Hiv Stigma, Internalized Homophobia, and Depression among Latino Msm Living with Hiv. Ann Behav Med 2012, 43:S277-S277.

28. Poteat VP, Scheer JR, Digiovanni CD, Mereish EH: Short-Term Prospective Effects of Homophobic Victimization on the Mental Health of Heterosexual Adolescents. J Youth Adolesc 2013.

29. Xu W, Zheng L, Xu Y, Zheng Y: Internalized homophobia, mental health, sexual behaviors, and outness of gay/bisexual men from Southwest China. International journal for equity in health 2017, 16(1):36.

30. Meyer IH, Frost DM: Minority stress and the health of sexual minorities. Handbook of psychology and sexual orientation 2013:252-266.

31. Rosario M, Schrimshaw EW, Hunter J: Disclosure of sexual orientation and subsequent substance use and abuse among lesbian, gay, and bisexual youths: critical role of disclosure reactions. Psychology of Addictive Behaviors 2009, 23(1):175.

32. Pollitt AM, Muraco JA, Grossman AH, Russell ST: Disclosure stress, social support, and depressive symptoms among cisgender bisexual youth. Journal of Marriage and Family 2017, 79(5):1278-1294.

33. Sivadon AD: Social support and smoking among Midwest LGBT adults. US: ProQuest Information \& Learning; 2015.

34. Liu H, Feng T, Ha T, Liu H, Cai Y, Liu X, Li J: Chinese culture, homosexuality stigma, social support and condom use: a path analytic model. Stigma research and action 2011, 1(1):27.

35. Cordero ED: Self-esteem, social support, collectivism, and the thin-ideal in Latina undergraduates. Body Image 2011, 8(1):82-85.

36. Cohen S, Wills TA: Stress, social support, and the buffering hypothesis. Psychol Bull 1985, 98(2):310.

37. Wong NY, Ahuvia AC: Personal taste and family face: Luxury consumption in Confucian and Western societies. Psychology and marketing 1998, 15(5):423-441.

38. Lai DW: Filial piety, caregiving appraisal, and caregiving burden. Research on Aging 2010, 32(2):200223. 
39. Koo F: The six categories of participation in physical activity among older Chinese Australians. Ageing Soc 2012, 1:67-84.

40. Li Y: Li Yihen Self Selections: Sex, Love, Marriage and Other Issues. In.: Hohhot: Inner Mongolia University Press; 2006.

41. Kong TS: Chinese male homosexualities: Memba, tongzhi and golden boy: Routledge; 2010.

42. Wah-Shan C: Homosexuality and the cultural politics of Tongzhi in Chinese societies. Journal of homosexuality 2001, 40(3-4):27-46.

43. Wang S, Song D, Huang W, He H, Wang M, Manning D, Zaller N, Zhang H, Operario D: Heterosexual Partnerships and the Need for HIV Prevention and Testing for Men Who Have Sex With Men and Women in China: A Qualitative Study. AIDS Education and Prevention 2015, 27(2):126-128.

44. Sun S, Budge S, Shen W, Xu G, Liu M, Feng SJSS, Medicine: Minority stress and health: A grounded theory exploration among men who have sex with men in China and implications for health research and interventions. Soc Sci Med 2020, 252:112917.

45. Liu P, Chan CS: Lesbian, gay and bisexual Asian American and their families. Asian Americans: Vulnerable populations, model interventions and clarifying agendas 2003:89-104.

46. Tao J, Ruan YH, Yin L, Vermund SH, Shepherd BE, Shao YM, Qian HZ: Sex with Women Among Men Who Have Sex with Men in China: Prevalence and Sexual Practices. Aids Patient Care St 2013, 27(9):524-528.

47. la Roi C, Meyer IH, Frost DM: Differences in sexual identity dimensions between bisexual and other sexual minority individuals: Implications for minority stress and mental health. American Journal of Orthopsychiatry 2019, 89(1):40.

48. Fish JN, Watson RJ, Gahagan J, Porta CM, Beaulieu-Prévost D, Russell ST: Smoking behaviours among heterosexual and sexual minority youth? Findings from 15 years of provincially representative data. Drug and alcohol review 2019, 38(1):101-110.

49. Peltzer K, Pengpid S: Minority stress among lesbian, gay, bisexual, and transgender (LGBT) university students in ASEAN countries: associations with poor mental health and addictive behavior. Gender and Behaviour 2016, 14(3):7806-7815.

50. Zheng L, Hart TA, Noor SW, Wen G: Stressors Based on Sexual Orientation and Mental Health Among Lesbian, Gay, and Bisexual Individuals in China: Minority Stress and Perceived Pressure to Get Married. Arch Sex Behav 2020.

51. Sun S, Pachankis JE, Li X, Operario D: Addressing minority stress and mental health among men who have sex with men (MSM) in China. Curr Hiv-Aids Rep 2020, 17(1):35-62.

52. Sun S, Budge S, Shen W, Xu G, Liu M, Feng S: Minority stress and health: A grounded theory exploration among men who have sex with men in China and implications for health research and interventions. Soc Sci Med 2020, 252:112917.

53. Shao J, Chang ES, Chen C: The relative importance of parent-child dynamics and minority stress on the psychological adjustment of LGBs in China. J Couns Psychol 2018, 65(5):598. 
54. Yu F, Nehl EJ, Zheng T, He N, Berg CJ, Lemieux AF, Lin L, Tran A, Sullivan PS, Wong FY: A syndemic including cigarette smoking and sexual risk behaviors among a sample of MSM in Shanghai, China. Drug Alcohol Depen 2013, 132(1-2):265-270.

55. He N, Wong FY, Huang ZJ, Thompson EE, Fu C: Substance use and HIV risks among male heterosexual and 'money boy' migrants in Shanghai, China. Aids Care-Psychological and SocioMedical Aspects of Aids/Hiv 2007, 19(1):109-115.

56. Feinstein BA, Dyar C: Bisexuality, minority stress, and health. Current sexual health reports 2017, 9(1):42-49.

57. Brewster ME, Moradi B, DeBlaere C, Velez BL: Navigating the borderlands: The roles of minority stressors, bicultural self-efficacy, and cognitive flexibility in the mental health of bisexual individuals. $J$ Couns Psychol 2013, 60(4):543.

58. Walters M, Chen, JT., Breiding, MJ.,: The National Intimate Partner and Sexual Violence Survey (NISVS): 2010 Findings on Victimization by Sexual Orientation. In. Atlanta, GA: National Center for Injury Prevention and Control, Centers for Disease Control and Prevention,; 2013.

59. Chinese Center for Disease Control and Prevention: Chinese Guidelines for Diagnosis and Treatment of HIV/AIDS (2018). Chin J Clin Infect Dis 2018, 11(6):411-432.

60. A Complete Guide to 2017 Minimum Wage Levels Across China [https://www.chinabriefing.com/news/complete-guide-2017-minimum-wage-levels-across-china/? hilite=\%27Guide\%27\%2C\%27Minimum\%27\%2C\%27Wages\%27\%2C\%27China\%27]

61. Radloff LS: The CES-D scale: a self-report depression scale for research in the general population. Applied psychological measurement 1977, 1(3):385-401.

62. Chaiton MO, Cohen JE, O'Loughlin J, Rehm J: A systematic review of longitudinal studies on the association between depression and smoking in adolescents. Bmc Public Health 2009, 9(1):356.

63. Song Y, Huang Y, Liu D, Kwan JSH, Zhang F, Sham PC, Tang SW: Depression in college: depressive symptoms and personality factors in Beijing and Hong Kong college freshmen. Comprehensive Psychiatry 2008, 49(5):496-502.

64. Cheung $\mathrm{C}-\mathrm{K}$, Bagley C: Validating an American scale in Hong Kong: the center for epidemiological studies depression scale (CES-D). The Journal of psychology 1998, 132(2):169-186.

65. Gonzales KL, Noonan C, Goins RT, Henderson WG, Beals J, Manson SM, Acton KJ, Roubideaux Y: Assessing the Everyday Discrimination Scale among American Indians and Alaska Natives. Psychological assessment 2016, 28(1):51.

66. Link BG: Understanding labeling effects in the area of mental disorders: An assessment of the effects of expectations of rejection. Am Sociol Rev 1987:96-112.

67. Testa RJ, Habarth J, Peta J, Balsam K, Bockting W: Development of the gender minority stress and resilience measure. Psychology of Sexual Orientation and Gender Diversity 2015, 2(1):65.

68. Martin JL, Dean L: Summary of measures: Mental health effects of AIDS on at-risk homosexual men. Unpublished manuscript 1987. 
69. Frost DM, Meyer IH: Internalized homophobia and relationship quality among lesbians, gay men, and bisexuals. J Couns Psychol 2009, 56(1):97.

70. Felitti VJ, Anda RF, Nordenberg D, Williamson DF, Spitz AM, Edwards V, Marks JS: Relationship of childhood abuse and household dysfunction to many of the leading causes of death in adults: The Adverse Childhood Experiences (ACE) Study. American journal of preventive medicine 1998, 14(4):245-258.

71. Prevalence of individual adverse childhood experiences [www.cdc.gov/violenceprevention/acestudy/prevalence.html]

72. Tonsing K, Zimet GD, Tse S: Assessing social support among South Asians: The multidimensional scale of perceived social support. Asian journal of psychiatry 2012, 5(2):164-168.

73. Campbell-Sills L, Stein MB: Psychometric analysis and refinement of the connor-davidson resilience scale (CD-RISC): Validation of a 10-item measure of resilience. Journal of Traumatic Stress: Official Publication of The International Society for Traumatic Stress Studies 2007, 20(6):1019-1028.

74. Connor KM, Davidson JR: Development of a new resilience scale: The Connor-Davidson resilience scale (CD-RISC). Depression and anxiety 2003, 18(2):76-82.

75. Thompson B: Exploratory and confirmatory factor analysis: Understanding concepts and applications. Applied Psychological Measurement 2007, 31(3):245-248.

76. Hu Lt, Bentler PM: Cutoff criteria for fit indexes in covariance structure analysis: Conventional criteria versus new alternatives. Structural equation modeling: a multidisciplinary journal 1999, 6(1):1-55.

77. Tuthill Z, Denney JT, Gorman B: Racial disparities in health and health behaviors among gay, lesbian, bisexual and heterosexual men and women in the BRFSS-SOP. Ethnicity \& health 2020, 25(2):177188.

78. Clarke MP, Coughlin JR: Prevalence of Smoking Among the Lesbian, Gay, Bisexual, Transsexual, Transgender and Queer (LGBTTQ) Subpopulations in Toronto - The Toronto Rainbow Tobacco Survey (TRTS). Canadian Journal of Public Health 2012, 103(2):132-136.

79. Normansell KM, Wisco BE: Negative interpretation bias as a mechanism of the relationship between rejection sensitivity and depressive symptoms. Cognition and Emotion 2017, 31(5):950-962.

80. Slavich GM, Thornton T, Torres LD, Monroe SM, Gotlib IH: Targeted rejection predicts hastened onset of major depression. Journal of social and clinical psychology 2009, 28(2):223-243.

81. Kittiteerasack P, Steffen A, Matthews A: The Influence of Minority Stress on Level of Depression among Thai LGBT Adults. Jurnal Keperawatan Indonesia 2020, 23(1):74-84.

82. Choi K-H, Steward WT, Miège P, Hudes E, Gregorich SE: Sexual Stigma, Coping Styles, and Psychological Distress: A Longitudinal Study of Men Who Have Sex With Men in Beijing, China. Arch Sex Behav 2015:1-9.

83. Pachankis JE, Hatzenbuehler ML, Starks TJ: The influence of structural stigma and rejection sensitivity on young sexual minority men's daily tobacco and alcohol use. Soc Sci Med 2014, 103:6775. 
84. Blosnich JR, Horn K: Associations of discrimination and violence with smoking among emerging adults: differences by gender and sexual orientation. Nicotine Tob Res 2011, 13(12):1284-1295.

85. Shires DA, Jaffee KD: Structural Discrimination is Associated With Smoking Status Among a National Sample of Transgender Individuals. Nicotine Tob Res 2016, 18(6):1502-1508.

86. Rosario M, Corliss HL, Everett BG, Russell ST, Buchting FO, Birkett MA: Mediation by peer violence victimization of sexual orientation disparities in cancer-related tobacco, alcohol, and sexual risk behaviors: Pooled youth risk behavior surveys. Am J Public Health 2014, 104(6):1113-1123.

87. Dong Wang: Bisexual identity: An in-depth interview [In Chinese]. Nanjing, China: Nanjing Normal University; 2013.

88. Ryan C, Huebner D, Diaz RM, Sanchez J: Family rejection as a predictor of negative health outcomes in white and Latino lesbian, gay, and bisexual young adults. Pediatrics 2009, 123(1):346.

89. Kosciw JG, Diaz EM: Involved, Invisible, Ignored: The Experiences of Lesbian, Gay, Bisexual and Transgender Parents and Their Children in Our Nation's K-12 Schools: ERIC; 2008.

90. Institute of Medicine: The Health of Lesbian, Gay, Bisexual, and Transgender People: Building a Foundation for Better Understanding. In. Washington, DC: National Academies Press; 2011.

\section{Figures}

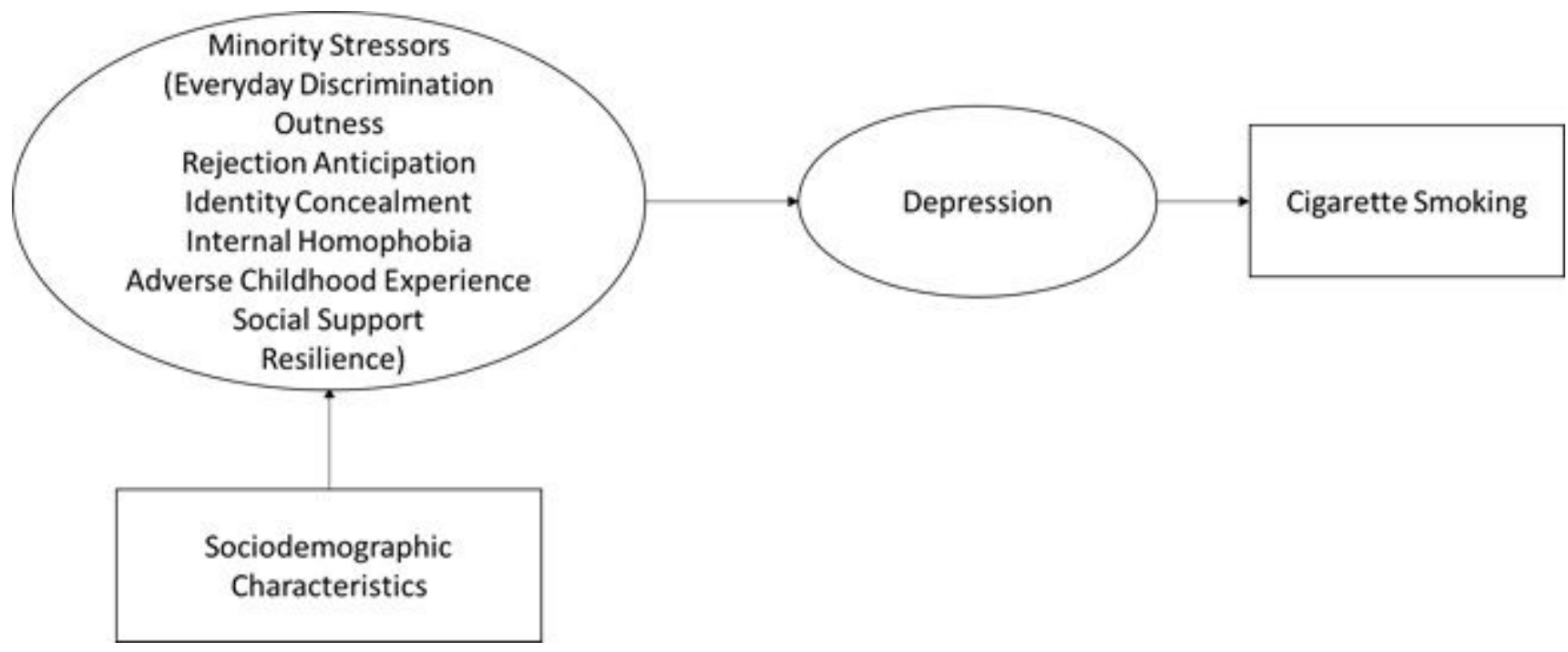

Figure 1

Hypothesized conceptual model of minority stressor to cigarette smoking among gay and bisexual men in China 


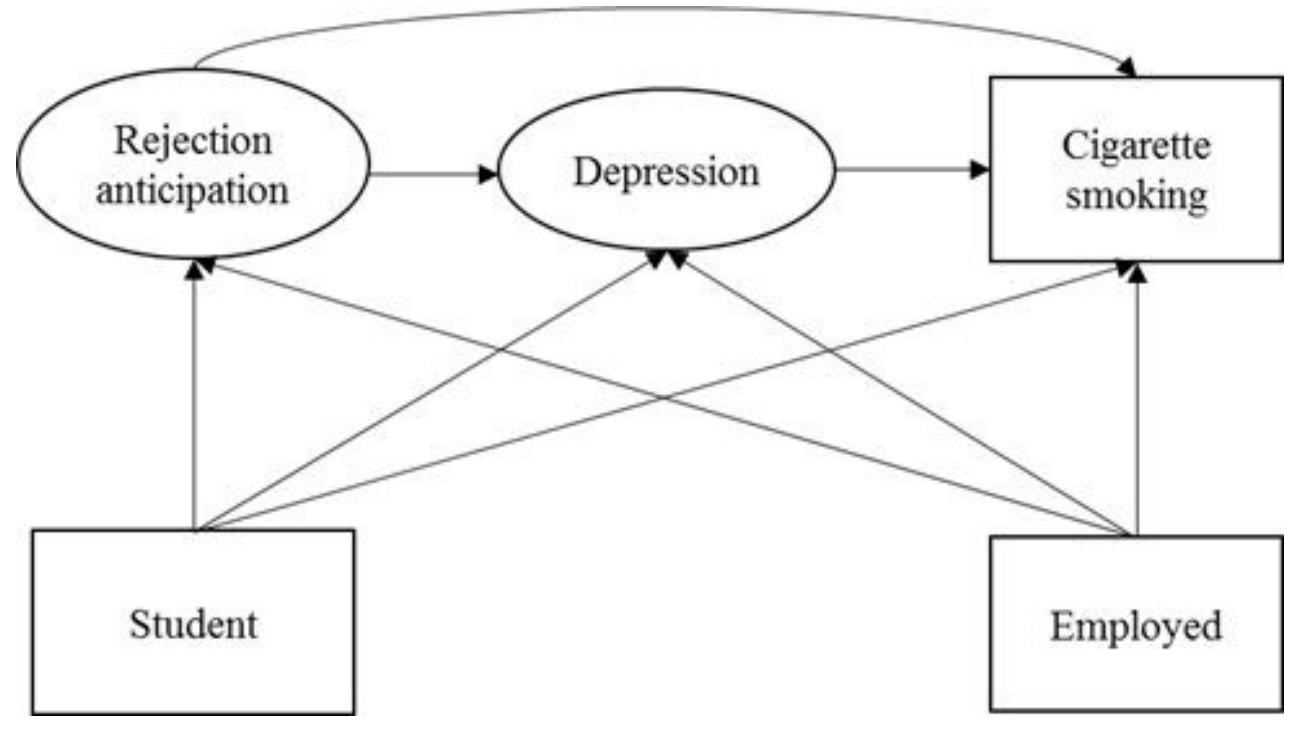

Figure 2

Specified two-group structural equation model of rejection anticipation to cigarette smoking for both gay and bisexual men in China.

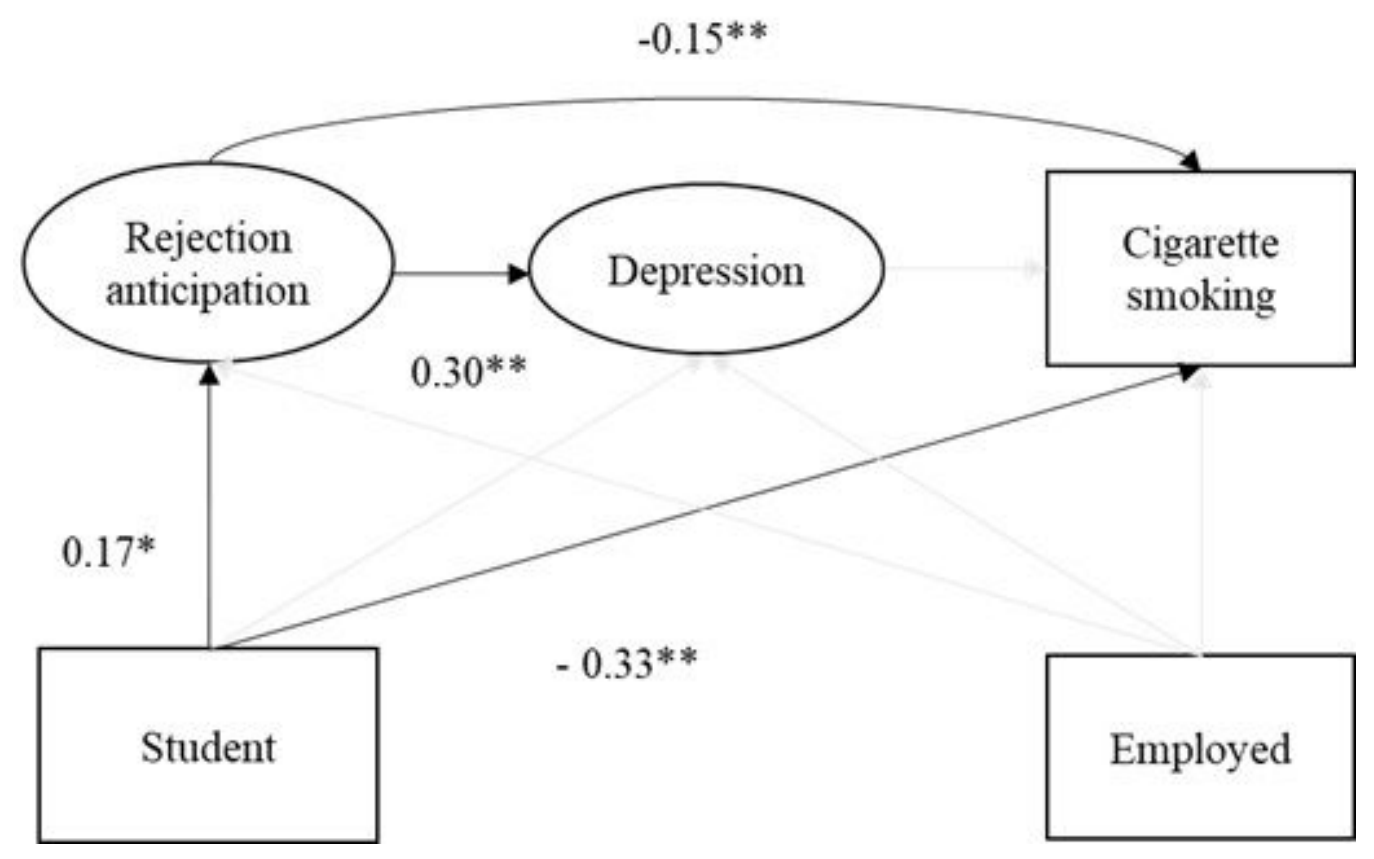

Figure 3

Final structural equation model testing pathway between rejection anticipation, depressive symptoms, and cigarette smoking among Chinese gay men and bisexual men. Model fit: $\chi 2=981.28, d f=770, p<0.001$ (recommended p>0.05); RMSEA: 0.028 (recommended <0.05); CFI: 0.920 (recommended >0.90); TLI: 0.916 (recommended >0.90); WRMR $=1.397$ (recommended < 1). Coefficients: Rejection anticipation was positively associated with greater depressive symptoms (standardized $\beta=0.30, p<.001$ ) and negatively associated with being a current cigarette smoker (standardized $\beta=-0.15, p<.001$ ). Being a student was positively associated with higher rejection anticipation (standardized $\beta=0.17, p<.05$ ) and was negatively 
associated with cigarette smoking (standardized $\beta=-0.33, p<.05$ ). Being a student was not associated with depressive symptoms. Depressive symptoms not associated with cigarette smoking. 